\title{
Nitrite: On the Journey from Toxin to Therapy
}

\author{
Arlin B. Blood · Gordon G. Power
}

Published online: 21 January 2015

(C) Springer International Publishing Switzerland 2015

Biomedical interest in the nitrite anion $\left(\mathrm{NO}_{2}{ }^{-}\right)$was once limited primarily to its toxic effects following ingestion of contaminated food or well water. The discovery that nitric oxide (NO) is an endogenously produced signaling molecule, and that nitrite is a relatively stable product of NO metabolism, brought increased focus on nitrite concentrations as an index of endogenous NO production. More recently, however, it has become apparent that nitrite is more than just a byproduct of NO, but that it can also be converted back into NO by a number of different biochemical pathways (see the recent review by Lundberg and Weitzberg [1]). As a result, nitrite is now widely regarded as a bioactive molecule with potentially wide-ranging physiological function and therapeutic application.

A majority of circulating nitrite is derived from the oxidation of NO produced by endothelial NO synthase [2]. A significant portion of circulating nitrite is also derived from plasma nitrate $\left(\mathrm{NO}_{3}{ }^{-}\right)$, which is concentrated $\sim 10$ fold from the plasma into the saliva where oral bacterial nitrate reductases efficiently reduce it to nitrite that is then swallowed and absorbed in the gastrointestinal tract [1].

Nitrite is metabolized in a number of different organs as well as in the blood, or excreted by the kidneys. When $\mathrm{O}_{2}$ is present, nitrite can be oxidized to produce nitrate. In contrast, under hypoxic conditions, nitrite can be reduced to NO that can then produce an array of nitrogen oxide species including nitrosothiols, $N$-nitrosamines, and iron-

\section{A. B. Blood ( $\square)$}

Division of Neonatology, Department of Pediatrics, Loma Linda University School of Medicine, Loma Linda, CA 92354, USA e-mail: ablood@1lu.edu

A. B. Blood - G. G. Power

Center for Perinatal Biology, Loma Linda University School of Medicine, Loma Linda, CA 92354, USA nitrosyls. Nearly all of these products can be converted back into nitrite again under physiological conditions, raising the possibility of bidirectional flux between nitrite and these other nitrogen oxide species (Fig. 1). The recently described reduction of nitrate $\left(\mathrm{NO}_{3}{ }^{-}\right)$to nitrite $\left(\mathrm{NO}_{2}{ }^{-}\right)$in the mouth, and nitrite to $\mathrm{NO}$ in the tissues constitutes a nitrate $\rightarrow$ nitrite $\rightarrow \mathrm{NO}$ axis that provides for NO production independent of the traditional NO synthase pathways. While the half-life of $\mathrm{NO}$ in whole blood ( $\sim 2 \mathrm{~ms}$ ) is so short that the effects of free NO are limited to a few hundred microns from its site of production, the elimination half-lives of nitrite (15-30 min) and nitrate (4-6 h) are long enough for these species to serve as circulating reservoirs of NO bioactivity. Thus, the widely different biologic half-lives provide responses by $\mathrm{NO}$ in seconds, nitrite as a substrate for NO production changing over minutes, and nitrate as a large-capacity reservoir changing over hours.

Most of the bioactivity of nitrite requires it first to be reduced to NO. This redox reaction can occur with a number of different proteins that carry transition metals such as hemoglobin, myoglobin, cytoglobin, and neuroglobin, other heme-containing proteins such as cytochrome c oxidase, and the molybdenum-containing proteins xanthine oxidase and aldehyde oxidase (see the recent review by Kim-Shapiro and Gladwin [3]). Importantly, when $\mathrm{O}_{2}$ binds to the transition metals of these proteins the reduction of nitrite to NO is blocked, and thus nitrite reduction to NO occurs only under hypoxic conditions when there is need for vasodilation and increased $\mathrm{O}_{2}$ delivery. This negative feedback system maintains $\mathrm{O}_{2}$ delivery without requiring neural or endocrine control. Nitrite can also be reduced to NO under acidic conditions in which it is protonated to form nitrous acid $\left(\mathrm{HNO}_{2}, \mathrm{pKa}=3.2\right)$ that then decomposes into NO in seconds. As a result, significant amounts 
Fig. 1 Circulating concentrations and half-lives of nitric oxide, nitrite, and nitrate. Nitric oxide is oxidized to nitrite, which is further oxidized to nitrate. Nitrate is reduced back into nitrite by commensal bacteria in the mouth, and nitrite is reduced to nitric oxide under hypoxic/ischemic conditions

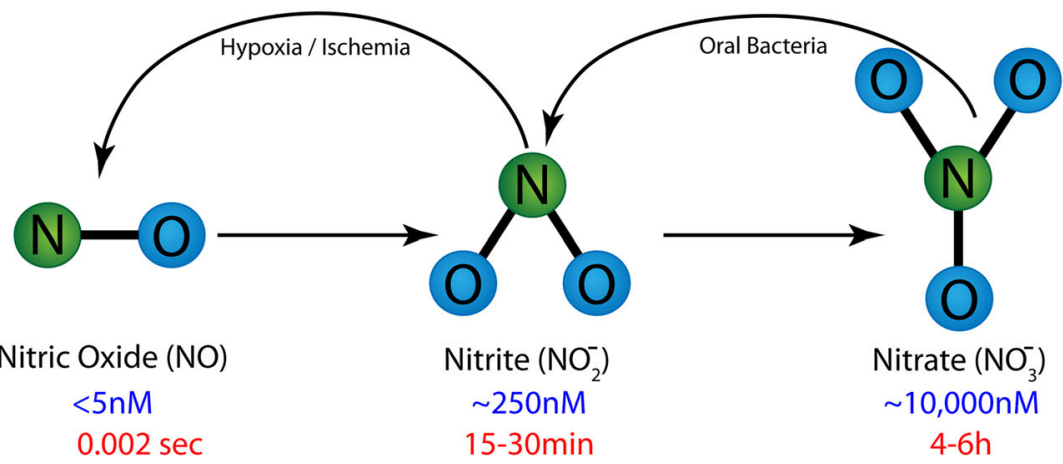

of NO can be derived from nitrite in the stomach and in severely ischemic/acidotic tissues, with the possibility of clinical relevance in myocardial ischemia, for example.

The NO bioactivity of nitrite opens the door to a wide array of potential clinical applications. In the lung, nitrite is being considered for the treatment of pulmonary hypertension. Inhaled nitrite aerosol is effective at reducing pulmonary hypertension caused by hypoxia or hemolysis in newborn lambs and adult humans [4-6]. Although the original mechanism proposed for this effect was the reduction of nitrite to $\mathrm{NO}$ by deoxyhemoglobin flowing into the lung [4], more recent studies indicate that nitrite may be converted to NO within the airway itself to effect the vasodilatory response [5]. In the current issue of this journal, Rix et al. [7] present the first human data for safety and pharmacokinetics of inhaled nitrite aerosol. In healthy volunteers, they find inhaled nitrite to be well tolerated within the expected therapeutic dose range. Systemic hypotension was the dose-limiting adverse effect. These results are consistent with the NO bioactivity of nitrite, in accord with earlier animal studies, and provide a rational basis for a trial of aerosolized nitrite in the treatment of pulmonary hypertension. Given the hypoxia-sensitive nature of NO production from nitrite, it is surprising that systemic hypoxia did not potentiate the hypotensive effects of nitrite, but this may have been due to a counteracting sympathetic response to the hypoxic stimulus. However, it is unknown in what form nitrite crosses the bronchial/ alveolar membranes. Other questions that remain include whether the transport is carrier mediated or $\mathrm{pH}$ dependent, what level of the vascular tree smooth muscle cells are relaxed, and whether this reduced smooth muscle tone is distributed throughout the lungs such that blood flow is more evenly matched with ventilation.

In addition to pulmonary hypertension, other potential uses of nitrite are being explored. Because the conversion of nitrite to $\mathrm{NO}$ is accelerated when $\mathrm{O}_{2}$ levels are low, much research has focused on nitrite's role in responses to ischemia/reperfusion stress. Modest increases in circulating nitrite concentrations prior to an ischemic insult confer marked protection against injury in many organ systems (see the review by Lundberg and Weitzberg [1]). The mechanism(s) for these protective effects appears to involve the reduction of nitrite to NO because the addition of NO scavengers is found to eliminate protection. Putative pathways for protection include the suppression of $\mathrm{O}_{2}$ consumption and lessened superoxide production during the ischemic insult due to $\mathrm{NO}$ competing with $\mathrm{O}_{2}$ for binding to the mitochondrial complex IV. In addition, the reduction of nitrite may result in $S$-nitrosation of a number of enzymes, ion channels, and transcription factors, resulting in functional changes that confer protection against ischemia and/or reperfusion injury [8]. Although the exact mechanism(s) of protection remain to be determined, supplemental nitrite is currently being studied in a number of indications including myocardial infarction, subarachnoid hemorrhage, cardiac arrest, organ transplant, and coronary bypass surgery.

Research over the past two decades has alerted us to the important contribution of nitrite to NO signaling. However, although the beneficial effects of dietary nitrite on exercise performance are already widely accepted [1] and the pharmaceutical use of nitrite is under investigation for a number of different indications, there is still much to learn regarding how such broad an effect is achieved by this anion we once thought to be physiologically irrelevant.

Acknowledgments This work was supported by a National Institues of Health grant HL095973.

Disclosure Gordon Power is a co-inventor on the patent for the therapeutic use of inhaled nitrite. Arlin Blood has no potential conflicts of interest that might be relevant to the contents of this manuscript.

\section{References}

1. Lundberg JO, Weitzberg E. NO-synthase independent NO generation in mammals. Biochem Biophys Res Commun. 2010;396(1): $39-45$.

2. Rhodes P, Leone AM, Francis PL, Struthers AD, Moncada S, Rhodes PM. The L-arginine: nitric oxide pathway is the major source of plasma nitrite in fasted humans. Biochem Biophys Res Commun. 1995;209(2):590-6. 
3. Kim-Shapiro DB, Gladwin MT. Mechanisms of nitrite bioactivation. Nitric Oxide. 2014;30(38):58-68.

4. Hunter CJ, Dejam A, Blood AB, Shields H, Kim-Shapiro DB, Machado RF, et al. Inhaled nebulized nitrite is a hypoxia-sensitive NO-dependent selective pulmonary vasodilator. Nat Med. 2004;10(10):1122-7.

5. Blood AB, Schroeder HJ, Terry MH, Merrill-Henry J, Bragg SL, Vrancken $\mathrm{K}$, et al. Inhaled nitrite reverses hemolysis-induced pulmonary vasoconstriction in newborn lambs without blood participation. Circulation. 2011;123(6):605-12.

6. Ingram TE, Pinder AG, Bailey DM, Fraser AG, James PE. Lowdose sodium nitrite vasodilates hypoxic human pulmonary vasculature by a means that is not dependent on a simultaneous elevation in plasma nitrite. Am J Physiol. 2010;298(2):H331-9.

7. Rix PJ, Vick A, Attkins NJ, Barker GE, Bott AW, Alcorn H, Jr., et al. Pharmacokinetics, pharmacodynamics, safety, and tolerability of nebulized sodium nitrite (AIR001) following repeat-dose inhalation in healthy subjects. Clin Pharmacokinet. 2014. doi:10. 1007/s40262-014-0201-y.

8. Rassaf T, Ferdinandy P, Schulz R. Nitrite in organ protection. Br J Pharmacol. 2014;171(1):1-11. 